\title{
NOUVELLE
}

\section{Le globule rouge}

\section{Un nouvel acteur dans la physiopathologie de la maladie de Gaucher}

Mélanie Franco, Caroline Le Van Kim
Physiologie du globule rouge normal et pathologique, Laboratoire d'excellence GR-Ex, Inserm U665, Université Paris-Diderot, Institut national de transfusion sanguine, 6, rue Alexandre Cabanel, 75739 Paris cedex 15, France. melanie.franco@inserm.fr caroline.le-van-kim@inserm.fr
> La maladie de Gaucher est une affection dont la prévalence se situe autour de 1/60000 dans la population générale. Cette maladie génétique rare est la plus commune des maladies lysosomales récessives. Elle est due à des mutations dans le gène GBA codant pour l'enzyme $\beta$-glucocérébrosidase (GBA) qui permet le catabolisme du glucosylcéramide. Ce défaut enzymatique conduit à l'accumulation de ce sphingolipide dans les lysosomes des monocytes et des macrophages. Il existe trois types de maladie de Gaucher; le type 1 touche plus de $90 \%$ des patients et est défini cliniquement par l'absence d'atteinte neurologique, contrairement aux types 2 et 3 . Les atteintes non neuronopathiques de la maladie de Gaucher sont viscérales (hépatosplénomégalie, infarctus splénique), hématologiques (thrombopénie, anémie) et osseuses. (es manifestations représentent les risques majeurs de la maladie, qui se caractérise notamment par une asthénie et des crises douloureuses invalidantes.

Depuis les années 1990, il existe un traitement spécifique efficace mais extrêmement coûteux de la maladie de Gaucher qui consiste en l'administration tous les 15 jours par voie intraveineuse de la GBA recombinante [1].

La physiopathologie de la maladie de Gaucher est mal connue et la gravité et l'évolution de la maladie sont très variables d'un patient à l'autre. Les macrophages surchargés en glucosylcéramide présentent une morphologie caractéristique des cellules de Gaucher et sont considérés comme les responsables de la symptomatologie, car ils infiltreraient le foie, la rate et la moelle osseuse. Le contexte inflammatoire et l'atteinte du système immunitaire seraient, eux aussi, secondaires à la dysfonction macrophagique [1].

Toutefois, des hypothèses récentes remettent en question le seul rôle des macrophages dans la maladie de Gaucher.

\section{Origine des crises vaso-occlusives}

de la maladie de Gaucher

Si l'on attribue à l'invasion de certains organes par les cellules de Gaucher les atteintes hématologiques et viscérales, en revanche, l'origine des infarctus osseux (crise osseuse douloureuse dans un contexte inflammatoire) reste inconnue. L'atteinte osseuse est également caractérisée par des signes de fragilité qui peuvent conduire à l'apparition de fractures pathologiques et sont un facteur de risque d'une nécrose avasculaire de la tête fémorale et des vertèbres [2]. Les crises vaso-occlusives peuvent être déclenchées par le ralentissement du flux sanguin dans la microcirculation, entraînant une ischémie, puis un infarctus tissulaire. Dans la maladie de Gaucher, ce type d'évènement peut survenir dans les os et la rate.

Or, les crises vaso-occlusives sont une des complications majeures de la drépanocytose, ce qui nous a incités à émettre I'hypothèse d'une certaine analogie entre ces événements vaso-occlusifs et ceux observés dans la maladie de Gaucher. La drépanocytose est une maladie génétique du globule rouge $(G R)$ due à une mutation du gène codant pour la $\beta$-globine. Cette mutation est responsable de la polymérisation de l'hémoglo- bine et de la falciformation des GR dans un environnement hypoxique. Associés aux propriétés rhéologiques et adhésives anormales des GR et à l'activation de l'endothélium vasculaire, ces évènements contribuent à l'obstruction des capillaires sanguins et à la survenue des crises de vaso-occlusion [3, 4]. Les complications ostéonécrotiques caractérisent ces deux pathologies très différentes et se ressemblent aux dires des cliniciens qui ont pu en observer chez les deux types de patients.

Des études antérieures avaient identifié des GR de morphologie anormale chez des patients atteints de la maladie de Gaucher ainsi qu'une accumulation de glucosylcéramide dans ces cellules [5, 6]. Nous avons émis I'hypothèse que dans cette maladie, les GR pourraient, comme dans la drépanocytose, intervenir dans la survenue des crises vaso-occlusives dans les os et la rate.

Nous avons étudié les propriétés des GR de sujets sains et les avons comparées à celles des GR d'une cohorte de patients atteints de maladie de Gaucher de type 1, non splénectomisés et ne recevant aucun traitement enzymatique. Nous avons analysé la morphologie des GR, leurs propriétés hémorhéologiques et leur adhérence à des cellules endothéliales dans des conditions reproduisant le flux physiologique dans les capillaires [7].

\section{Propriétés anormales des globules} rouges de patients atteints de maladie de Gaucher

Chez les patients, une proportion plus élevée de GR présentaient des anomalies morphologiques en comparaison de ceux 
A

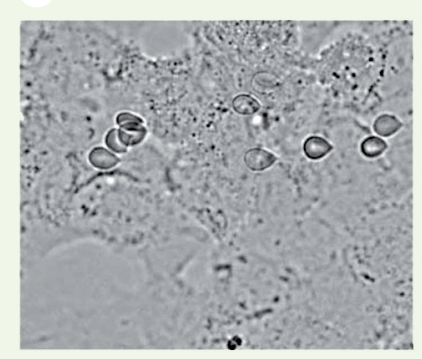

Gau

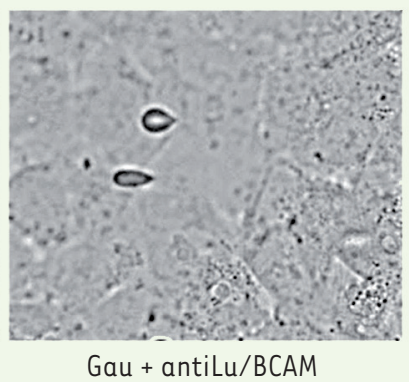

B

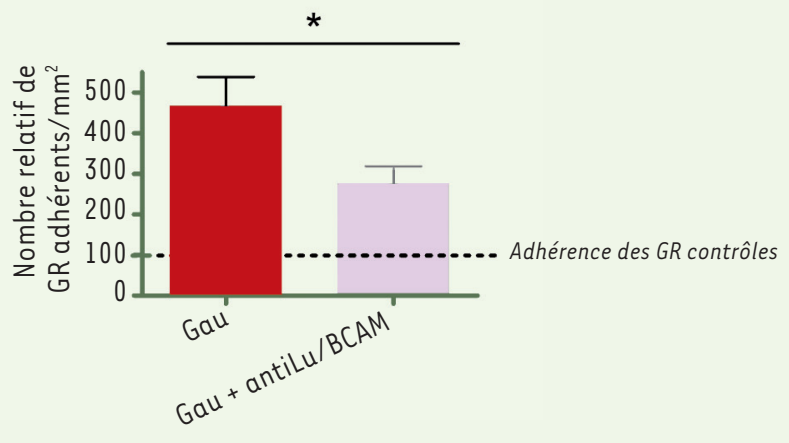

Figure 1. La molécule d'adhérence Lu/BCAM est impliquée dans l'adhérence des GR aux cellules endothéliales HMEC -1. A. Images représentatives montrant que les GR Gaucher (Gau) sont plus adhérents sur une monocouche de HMEC-1 que ceux préalablement incubés avec un anticorps dirigé contre la protéine Lu/BCAM (Gau + antiLu/BCAM). B. Nombre relatif de globules rouges $/ \mathrm{mm}^{2}$ adhérents aux HMEC-1 suite à une perfusion puis lavage à une vitesse de cisaillement de 2 dynes $/ \mathrm{cm}^{2}$. Chaque condition a été testée à partir de prélèvements sanguins issus de deux patients atteints de la maladie de Gaucher ( $p<0,05 *$, test Mann Whithey).

des individus sains. L'étude hémorhéologique a mis en évidence un défaut d'élasticité membranaire qui se traduit par une agrégation augmentée, ainsi qu'une déformabilité diminuée des GR isolés chez des patients atteints de maladie de Gaucher comparés aux GR contrôles. La viscosité sanguine est également altérée chez ces patients. Les expériences d'adhérence en flux dynamique ont montré une augmentation significative de l'adhérence des GR de patients à des cellules endothéliales microvasculaires humaines (HMEC-1) et à la laminine 511/521, un composant majeur de la matrice extracellulaire (Figure 1).

La molécule d'adhérence Lu/BCAM (Lutheran/basal cell adhesion molecule), membre de la superfamille des immunoglobulines, est le récepteur unique de la laminine 511/521 dans les GR et les progéniteurs érythroïdes. $0 r$, il a été montré que l'activation de Lu/BCAM, soit par phosphorylation dans les GR drépanocytaires, soit par dissociation du squelette membranaire dans les GR sphérocytaires ${ }^{1}$, induisait une

\footnotetext{
${ }^{1}$ La sphérocytose héréditaire est une anémie hémolytique constitutionnelle liée à la présence de GR de forme sphérique (contrairement à la forme biconcave habituelle) sur le frottis sanguin, et secondaire à une anomalie génétique d'une des protéines de la membrane du GR (spectrine, ankyrine, protéine 4.2 , bande 3 ).
}

adhérence anormale des GR à la laminine $[8,9]$. De manière similaire, nous avons montré que Lu/BCAM est surexprimée et activée par phosphorylation dans les GR de patients atteints de maladie de Gaucher.

L'ensemble de ces données mettent en évidence des anomalies membranaires des GR dans la maladie de Gaucher, une activation de Lu/BCAM et une adhérence augmentée à l'endothélium vasculaire (Figure 2). L'état proadhérent de ces GR, combiné aux défauts hémorhéologiques, sont des facteurs pouvant contribuer aux évènements vaso-occlusifs.

La maladie de Gaucher est une maladie de surcharge lipidique dans laquelle les glucocérébrosides et autres lipides dérivés s'accumulent dans les cellules. Notre hypothèse est que l'accumulation de ces lipides dans les érythrocytes perturberait la composition lipidique des membranes ainsi que la distribution latérale des protéines à la surface des GR, conduisant à l'expression et à l'activation anormales de molécules d'adhérence comme Lu/BCAM [7].

Une « érythrocytopathie » jusqu'alors négligée

La maladie de Gaucher est décrite comme une glucosphingolipidose des macrophages; l'accumulation de lipides proviendrait des GR et des leucocytes que ces macrophages phagocytent. II est admis que les macrophages sont les premières cellules touchées dans la maladie. Nous avons mis en évidence chez ces patients la présence de GR possédant des propriétés anormales, ce qui pourrait stimuler leur propre destruction par les macrophages. Les GR pourraient être ainsi les premières cellules atteintes dans la maladie de Gaucher, et elles conduiraient, secondairement et par la phagocytose qu'induisent leurs anomalies, à la formation de cellules de Gaucher macrophagiques dans la rate.

\section{Conclusion}

La maladie de Gaucher n'est pas uniquement une glucosphingolipidose des macrophages mais s'avère être aussi une érythrocytopathie jusqu'alors négligée et qui serait directement impliquée dans l'étiologie de la maladie. Les anomalies des GR identifiées dans cette maladie, pourraient contribuer aux évènements vaso-occlusifs observés dans les os et la rate. Une hypothèse séduisante serait que l'évènement originel dans la physiopathologie de la maladie de Gaucher pourrait être un défaut des érythrocytes entraînant leur phagocytose accélérée par les macrophages (Figure 2). Nous avons également montré la présence 


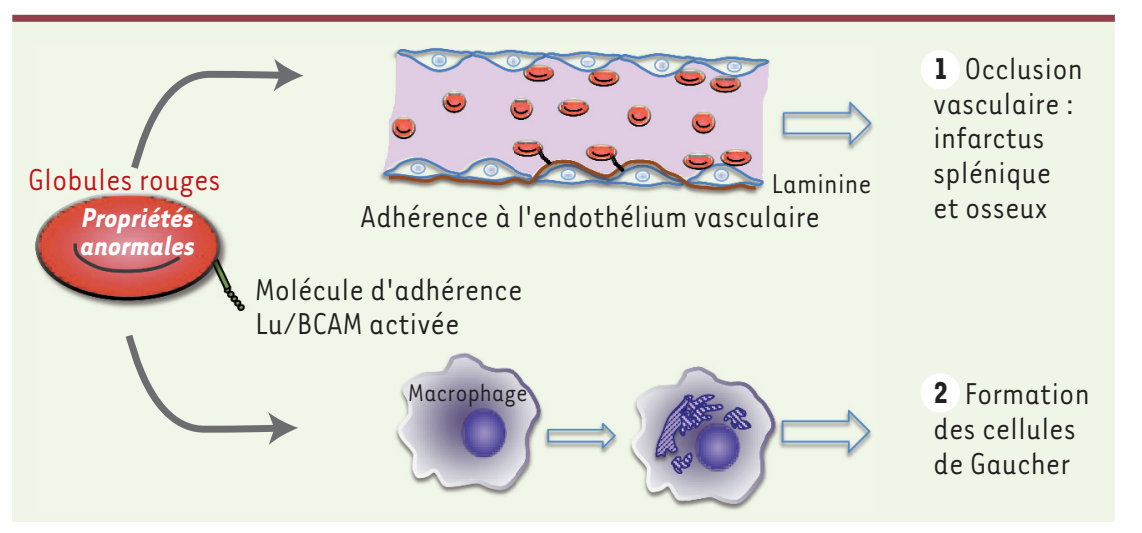

active de GBA dans les progéniteurs érythroïdes précoces mais pas dans les GR matures [7]. Ainsi, I'accumulation lipidique observée dans les GR pourrait survenir dès le stade de progéniteur érythroïde. Toutefois, de forts taux de glucosylcéramide sont observés dans le plasma des patients, laissant penser qu'il pourrait aussi s'agir d'une accumulation passive de ces lipides [10]. Ces deux hypothèses seront évaluées dans nos travaux futurs.

En conclusion, les $G R$, et plus particulièrement leur propriété d'adhérence, représentent des acteurs clés dans la physiopathologie de nombreuses maladies hématologiques et non hématologiques qui ont en commun d'être associées à des événements thrombotiques ou vaso-occlusifs non expliqués. $\diamond$

Red blood cell, a new player

in the pathophysiology of

Gaucher disease

\section{REMERCIEMENTS}

Nous remercions Emmanuel Collec et Philippe Connes pour leur contribution à l'étude des propriétés anormales des globules rouges dans la maladie de Gaucher. Nous remercions également les Dr Cyril Mignot, Nadia Belmatoug et le Pr Thierry Billette de Villemeur pour leur collaboration et l'accès aux patients. Le projet a été en partie financé par le programme d'investissement d'Avenir (ANR, ANR-11LABX-0051; ANR-11-IDEX-0005-02) et Shire France.

\section{LIENS D'INTÉRÊT}

Les auteurs déclarent n'avoir aucun lien d'intérêt concernant les données publiées dans cet article.

\section{RÉFÉRENCES}

1. Cox TM, Schofield JP. Gaucher's disease: clinical features and natural history. Baillieres Clin Haematol 1997 ; $10: 657-89$.

2. Stowens DW, Teitelbaum SL, Kahn AJ, Barranger JA. Skeletal complications of Gaucher disease. Medicine $1985 ; 64: 310-22$.
Figure 2. Rôles potentiels des globules rouges dans la physiopathologie de la maladie de Gaucher. Les anomalies morphologiques, rhéologiques et l'activation de molécules d'adhérence comme Lu/BCAM suggèrent un rôle important des globules rouges : (1) dans les occlusions vasculaires pouvant s'expliquer par leur adhérence anormale à l'endothélium; (2) dans la formation des cellules de Gaucher lors de l'érythrophagocytose par les macrophages.

3. Cartron JP, Elion J. Erythroid adhesion molecules in sickle cell disease: effect of hydroxyurea. Transf Clin Biol 2008 ; 15 : 39-50.

4. Nash GB, Johnson CS, Meiselman HJ. Rheologic impairment of sickle RBCs induced by repetitive cycles of deoxygenation-reoxygenation. Blood $1988 ; 72$ : 539-45.

5. Bratosin D, Tissier JP, Lapillonne H, et al. A cytometric study of the red blood cells in Gaucher disease reveals their abnormal shape that may be involved in increased erythrophagocytosis. Cytometry B Clin Cytom $2011 ; 80: 28-37$.

6. Nilsson 0 , Hakansson G, Dreborg S, et al. Increased cerebroside concentration in plasma and erythrocytes in Gaucher disease: significant differences between type I and type III. Clin Genet $1982 ; 22: 274-9$.

7. Franco M, Collec $\varepsilon$, Connes P, et al. Abnormal properties of red blood cells suggest a role in the pathophysiology of Gaucher disease. Blood 2013; $121: 546-55$.

8. Gauthier $\varepsilon$, દl Nemer W, Wautier MP, et al. Role of the interaction between Lu/BCAM and the spectrinbased membrane skeleton in the increased adhesion of hereditary spherocytosis red cells to laminin. $\mathrm{Br}$ J Haematol 2010 ; 148 : 456-65.

9. Bartolucci P, Chaar V, Picot J, et al. Decreased sickle red blood cell adhesion to laminin by hydroxyurea is associated with inhibition of Lu/BCAM protein phosphorylation. Blood $2010 ; 116: 2152-9$.

10. Groener JE, Poorthuis BJ, Kuiper S, et al. Plasma glucosylceramide and ceramide in type 1 Gaucher disease patients: correlations with disease severity and response to therapeutic intervention. Biochem Biophys Acta 2008 ; 1781 : $72-8$.

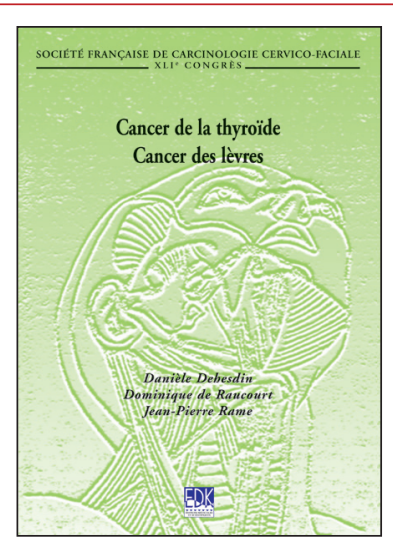

ISBN : 978-2-8425-4137-8 264 pages
Bon de commande à retourner à EDK, 25, rue Daviel - 75013 Paris Tél. : 0158101905 - Fax : 0143293262 - E-mail : edk@edk.fr NOM : Prénom : Adresse :

Code postal : Ville :

Pays :

Fonction :

Je souhaite recevoir l'ouvrage Cancer de la thyrö̈de - Cancers des lèvres : $35 €+3 €$ de port $=\mathbf{3 8} €$ TTC en ................ exemplaire, soit un total de ......................................... €

$\square$ Par chèque, à l'ordre de $\mathbf{E} \mathbf{D} \mathbf{K}$

$\square$ Par carte bancaire : $\square$ Visa $\square$ Eurocard/Mastercard

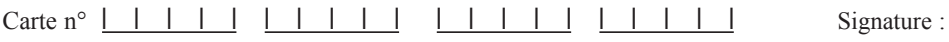

Date d'expiration: $\quad 1 \quad 1 \quad 1 \quad 1 \quad$

$\mathrm{N}^{\circ}$ de contrôle au dos de la carte : 